\title{
Waters in hydrothermal and polar environments: further developments for Si isotope measurements
}

\author{
SOPHIE OPFERGELt, FranÇOIS GASPARD, CATHERINE \\ HIRST \\ Earth and Life Institute, Université catholique de Louvain, \\ Louvain-la-Neuve, Belgium \\ (sophie.opfergelt@uclouvain.be)
}

The number of developed "non-traditional stable isotope systems" (non-CHONS) has gradually increased over the past 20 years. Some of the earliest systems, such as $\mathrm{Si}$ isotopes $\left(\delta^{30} \mathrm{Si}\right)$, have been applied in planetary, terrestrial and marine environments where measurements on rocks, soils or Siaccumulating plants were not limited by the $\mathrm{Si}$ amount available for analysis. For seawater (with low $\mathrm{Si}$ concentration), a pre-concentration method was set up. Adaptations were also made to allow $\delta^{30} \mathrm{Si}$ measurement on waters with (i) low anions/Si ratios (using external anion doping), or (ii) dissolved organic carbon (either with low DOC concentrations, or with high available $\mathrm{Si}$ amount to allow a burning step).

The challenge is now to go beyond existing applications in environments with more complex water matrices (such as high anions/Si ratios acidic hydrothermal environments and organic-rich peatlands), or in environments with low $\mathrm{Si}$ concentrations (such as polar environments at snowmelt).

We developed sample preparation techniques adapted for acidic and anion-rich water samples, for organic-rich water samples, and for water samples limited in Si amount, for which the classically used pre-concentration method would not work. Depending on the sample size and sample matrix, adapted sample preparation protocols have been established to add to the commonly used cation exchange column: either a preconcentration, a treatment for organics, and/or an anion exchange column. Checking Si recovery, and removal of cations, anions and organics is needed to prevent mass bias and produce reproducible MC-ICP-MS measurements.

These developments open new avenues for $\delta^{30} \mathrm{Si}$ measurements on water samples previously considered as poorly suited for $\delta^{30} \mathrm{Si}$ analysis, and for more collaborative work on archived water samples. Riverine Si fluxes are linked to the carbon cycle via silicate weathering and diatom $\mathrm{Si}$ uptake. Such analytical developments allow for the inclusion of challenging hydrothermal and polar environments in the collective effort to connect Long Term Ecological Research (LTER) network and Critical Zone Observatories (CZO) to assess how riverine $\mathrm{Si}$ export respond to global change. 